\title{
Feasibility of Laser Induced Plasma Micro-machining (LIP-MM)
}

\author{
Kumar Pallav and Kornel F. Ehmann \\ Department of Mechanical Engineering \\ Northwestern University \\ Evanston, IL 60208, USA \\ kumarpallav2008@u.northwestern.edu, k-ehmann@northwestern.edu
}

\begin{abstract}
The paper offers evidence on the feasibility of a newly proposed micromachining process, which is motivated by the need to overcome the various limitations associated with $\mu$-EDM and conventional ultra-short laser micromachining processes. The limitations in $\mu$-EDM and laser micro-machining processes are mainly due to the requirement of a conductive electrode and workpiece, electrode wear and compensation strategies, and complex process control mechanisms respectively. The new process uses a laser beam to generate plasma in a dielectric near the workpiece surface whose explosive expansion results in material removal by mechanisms similar to those that occur in $\mu$-EDM.
\end{abstract}

Keywords: Laser-machining, Micro-machining, $\mu$-EDM, Plasma.

\section{Introduction}

Micro-manufacturing encompasses the creation of high precision 3D products with feature sizes ranging from a few microns to a few millimeters. While micro-scale technologies are well established in the semiconductor and micro-electronics fields, the same cannot be said for manufacturing products involving highly accurate complex 3D geometries in materials such as metals, ceramics and polymers.

Micro-EDM ( $\mu$-EDM) has been one of the frontrunners for material removal in the aforementioned size range. Material removal is facilitated by the plasma created when the electric field generated due to high voltage between the electrode and the workpiece exceeds the threshold value for dielectric breakdown. The created plasma explosively expands and sends shock waves to the surface of the workpiece at very high speed that lead to localized heating and ablation. It has been widely recognized that the major limitations of $\mu-E D M$ are [1-3]: (1) the inability to micro-machine nonconductive materials and metals with low conductivity, (2) non-productive time and cost associated with on-machine electrode manufacturing, (3) frequent electrode replacements due to high electrode wear, (4) flushing of debris from the small electrode-workpiece gap $(<5 \mu \mathrm{m})$, and (5) low MRR $\left(0.6-6 \mathrm{~mm}^{3} / \mathrm{hr}\right)$.

A competing process is ultra-short laser pulse-based micro-machining. Ultra-short pulsed lasers have pulse durations ranging from (ps) to few (fs) at pulse frequencies ranging up to $500 \mathrm{kHz}$, and very low average power (up to $10 \mathrm{~W}$ ). However, since 
pulse duration is very short, the peak power is very high [4, 5]. With ultra-short laser pulses, the ablation process is dependent on a wide number of parameters including the type of polarization, type of material, peak pulse power, pulse repetition rate, pulse duration, environmental conditions and pressure, and wavelength. The ablation process is also accompanied by electron heat conduction and formation of a molten zone inside the metal target which reduces precision when machining metals [7]. The dependence of the ablation process on so many parameters makes conventional ultrashort laser micro-machining a very complex process and controlling all of these parameters is a major challenge [7].

The new micro-machining process described in this paper is motivated by the need to overcome the limitations of $\mu$-EDM and of conventional ultra-short laser micromachining. The new micro-machining process, to be termed Laser Induced Plasma Micro-machining (LIP-MM) uses an alternative mechanism for plasma creation to facilitate material removal.

\subsection{The Laser-Induced Plasma Micromachining (LIP-MM) Process}

The underlying principle of the proposed LIP-MM process rests on the fact that when an ultra-short pulsed laser beam is tightly focused in a transparent dielectric medium, extremely high peak power densities are reached at the focal spot $[6,8,9]$. And, when the peak power density exceeds the threshold irradiance required for dielectric breakdown, rapid ionization of the medium occurs leading to plasma formation. The plasma formed is optically opaque and causes a drastic increase in the absorption coefficient, which, in turn, gives rise to a rapid energy transfer from the radiation field to the medium $[6,8]$. This process is, in essence, an optical breakdown when the free electron density at the focal spot exceeds the critical value of $10^{20} \mathrm{~cm}^{-3}$. At this value, the plasma absorbs nearly all of the incoming laser beam's energy leading to rapid heating of the material in the focal volume [9]. The plasma leads to a liquid-gas phase change and causes cavitation accompanied by the generation of shock waves. If the focal volume is slightly above the surface of the workpiece, the explosive expansion of the plasma that is followed by the generation of shock waves, like in the case of a $\mu$-EDM discharge, results in material removal.

Plasma generation by ultra-short pulsed lasers, as described above does not require a conductive electrode and workpiece [9]. Actually, there is no need for an electrode. Even more importantly, the plasma's properties can be controlled by controlling the laser process parameters. Hence, many of the limitations of $\mu$-EDM and conventional laser micro-machining can be potentially circumvented through the LIP-MM process. In the subsequent sections initial results aimed at confirming the viability of the proposed material removal mechanism are presented.

\section{Experimental Setup and Results}

For the experimental realization of the LIP-MM process, a commercial, Nd:YVO laser system (Lumera laser, Rapid) emitting laser pulses of 8 ps pulse duration at a $532 \mathrm{~nm}$ wavelength was used. The beam profile of the (ps) pulses was Gaussian $\left(\mathrm{TEM}_{00}\right)$ and the beam was linearly polarized. The pulse repetition rate for the laser 
system varied from $10 \mathrm{kHz}$ to $500 \mathrm{kHz}$. For plasma generation, since very high peak power densities are required, low repetition rates ranging from $10 \mathrm{kHz}$ to $50 \mathrm{kHz}$ were used. The laser machine used in the experiments is capable of producing a peak power density level of several $10^{11} \mathrm{~W} / \mathrm{cm}^{2}$ at a spot size of $21 \mu \mathrm{m}$ in diameter. It is also equipped with a 5-axis programmable motion controller that directs the beam. The system has a resolution of $0.01 \mu \mathrm{m}$ and feed rates ranging from $0.01 \mu \mathrm{m} / \mathrm{s}$ to $10 \mathrm{~cm} / \mathrm{s}$, allowing a very precise control of the relative motion between the beam/plasma and the workpiece.

The laser beam was brought to the focusing lens through a beam delivery system consisting of various mirrors and a $3 \mathrm{X}$ beam expander. The beam expander expands the beam diameter from $0.6 \mathrm{~mm}$ to $1.8 \mathrm{~mm}$ before it reaches the focusing triplet lens. The focusing triplet lens focuses the laser beam to a diffraction limited spot size of 21 $\mu \mathrm{m}$ at the focal spot. Water was selected as the dielectric for the LIP-MM process. The laser pulses were focused into a glass beaker filled with water. Figure 1 shows the experimental setup used in the LIP-MM process.

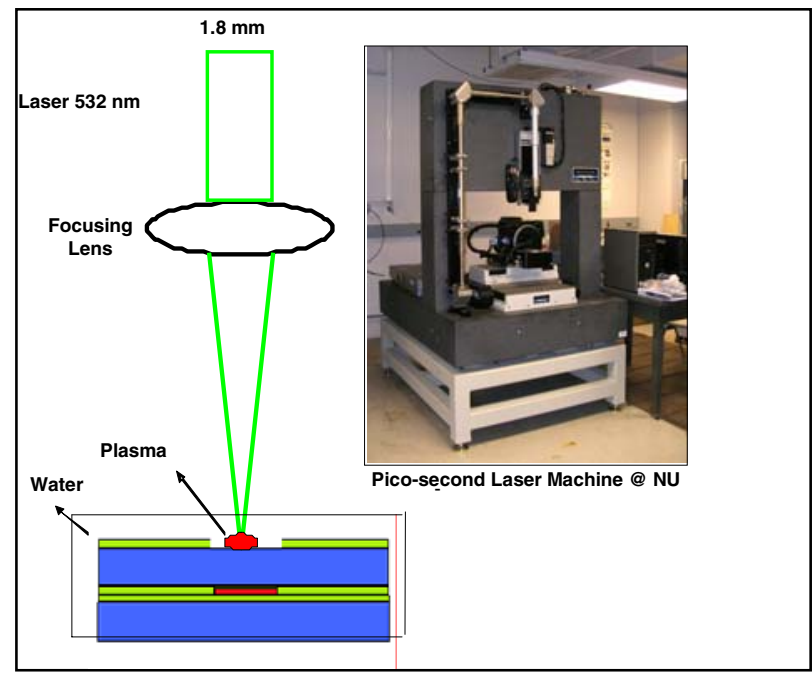

Fig. 1. Schematics of the LIP-MM Process

\subsection{Determination of Breakdown Threshold and Plasma Occurrence Probability}

The peak power density reached at the focal spot with ultra-short lasers generally ranges from several $10^{9} \mathrm{~W} / \mathrm{cm}^{2}$ to few $10^{11} \mathrm{~W} / \mathrm{cm}^{2}$. Ionization starts as soon as the peak power density exceeds the threshold ionization potential for the dielectric medium that depends on a number of factors including: laser wavelength, laser and dielectric media interaction time, and additional conditions such as the number of seed electrons, the type of dielectric, and the type of polarization of the laser beam [9]. Dielectric breakdown resulting in plasma generation can only be achieved when the peak power density exceeds the threshold value required to initiate ionization [6]. 
For plasma generation and control, the breakdown threshold corresponding to different pulse repetition rates was determined by imaging the plasma formed with a CCD camera (Edmund Optics, Guppy F-146 FireWire A 1/2" CCD Monochrome Camera) with a 2.5 - 10X magnification (Edmund Optics, VZM 1000i Video Lens) as the peak power density was varied from superthreshold to subthreshold values. During each laser exposure, the output average power of the incident laser irradiation was measured using an external laser power meter (Gentec SOLO 2 (R2)). Plasma generation due to dielectric breakdown was visually detected in a darkened room with the CCD camera and was recorded at a frame rate of 15 frames per second. Figure 2 shows the plasma images obtained through the CCD camera at different average power levels and pulse repetition rates. For each peak power density value at every pulse repetition rate, at least 20 laser exposures were evaluated for determining the plasma occurrence probability.

As shown in Fig. 2, with a decrease in peak power density levels at all repetition rates, the intensity of the plasma image decreases until it reaches the dielectric ionization threshold value where the plasma occurrence probability drops to below $10 \%$ and the plasma image cannot be obtained through the CCD camera. The mean breakdown threshold intensity corresponding to $10 \%$ plasma occurrence probability was experimentally measured to be $1.10 \times 10^{11} \mathrm{~W} / \mathrm{cm}^{2}$. However, the plasma size remained independent of the average power levels at all pulse repetition rates. Figure 3 shows the dependence of the plasma occurrence probability at different pulse repetition rates on peak power density. It shows that the breakdown threshold in water, corresponding to $10 \%$ plasma occurrence probability is approximately constant for all pulse repetition rates.
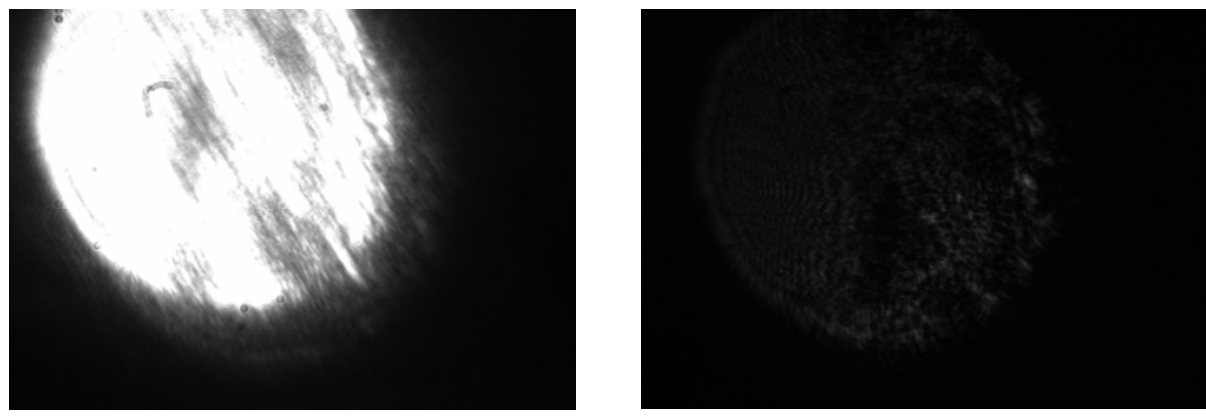

Fig. 2. Plasma Images at $10 \mathrm{kHz}, 63 \mathrm{~mW}$ and $38 \mathrm{~mW}$ Average Power Levels

\subsection{Laser Induced Plasma Micro-machining of Steel and Quartz}

For making micro channels in steel using the LIP-MM process, a pulse repetition rate of $10 \mathrm{kHz}$ and a peak power density corresponding to a $70 \%$ plasma occurrence probability were selected. The laser beam traverse speed was kept at two levels, i.e., at 100 $\mu \mathrm{m} / \mathrm{sec}$ and $500 \mu \mathrm{m} / \mathrm{sec}$ for machining single pass channels. 


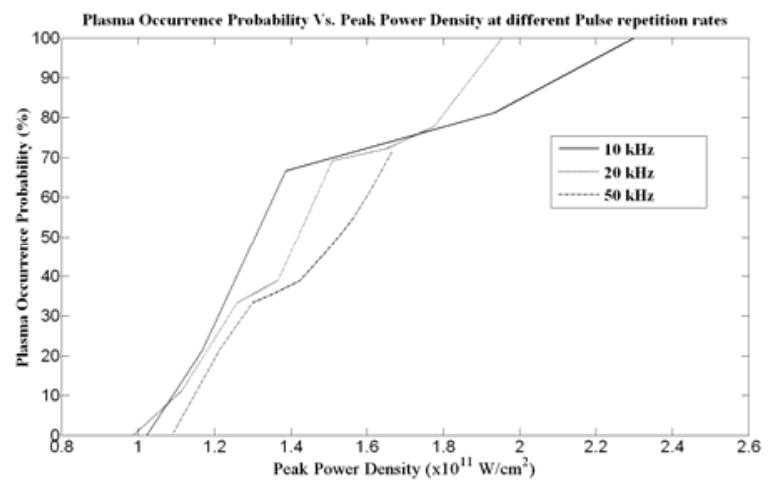

Fig. 3. Plasma Occurrence Probability at Different Peak Power Densities and Pulse Repetition Rates

Figure 4 shows the image of a single pass channel made in steel with the LIPMM process at a laser beam traverse speed of $100 \mu \mathrm{m} / \mathrm{sec}$ and peak power density corresponding to $70 \%$ plasma occurrence probability at a $10 \mathrm{kHz}$ pulse repetition rate. The channel has an approximately $30 \mu \mathrm{m}$ depth at various cross-sections and the walls of the channel are not vertical. The depths of the micro-machined channels were examined at various locations through a white light interferometer (Zygo NewView 7300). It was observed that the depth of the channels varied inversely with variations in the laser beam traverse speed. At a lower laser beam traverse speed of $100 \mu \mathrm{m} / \mathrm{s}$, the depth of the channel was approximately $30 \mu \mathrm{m}$, whereas with the higher laser beam traverse speed of $500 \mu \mathrm{m} / \mathrm{s}$, the depth of the channel was only $6 \mu \mathrm{m}$.
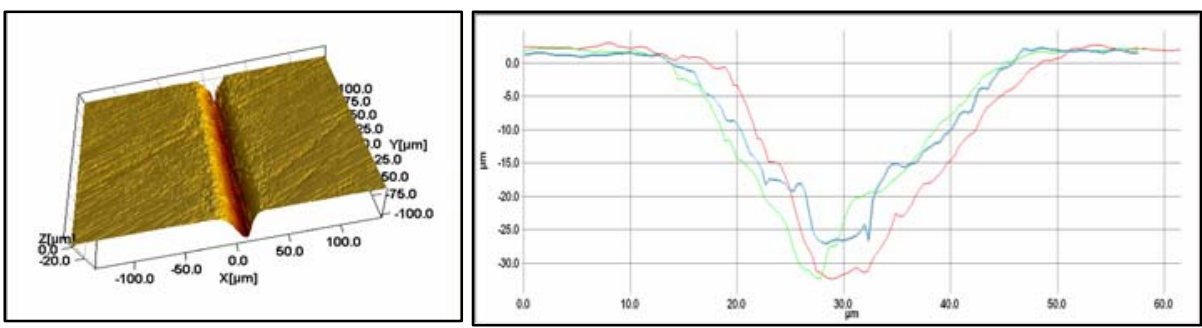

Fig. 4. Channels Made by LIP-MM in Steel at an Average Power of $59 \mathrm{~mW}$, Pulse Repetition Rate of $10 \mathrm{kHz}$ and Laser Beam Traverse Speed of $100 \mu \mathrm{m} / \mathrm{s}$

Figure 5 shows the images obtained through a SEM (Hitachi S3400N II) of channels machined in steel with different numbers of passes. The other process parameters were similar to the channel shown in Fig. 4. 

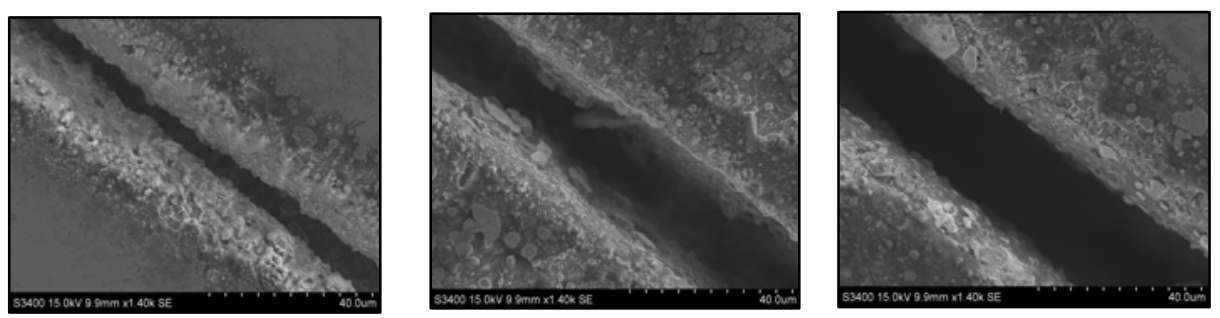

Fig. 5. SEM Images of Channels Made in Steel Through LIP-MM Process with 1,5 and 10 Passes, $10 \mathrm{kHz}$ Pulse Repetition Rate, Peak Power Density Level Corresponding to 70\% Plasma Occurrence Probability and Laser Beam Traverse Speed of $100 \mu \mathrm{m} / \mathrm{s}$

As shown in Fig. 5, with single pass micro-machining the channel had a significant recast layer and inclined walls. However with an increase in the number of passes, the walls became vertical and the recast layer decreased significantly. The depth of the channel became more uniform and also increased with an increase in the number of passes. Hence, with an increase in the number of passes, the surface features of the channel improve due to the vertical walls, uniform depth, and significant reduction in the recast layer.

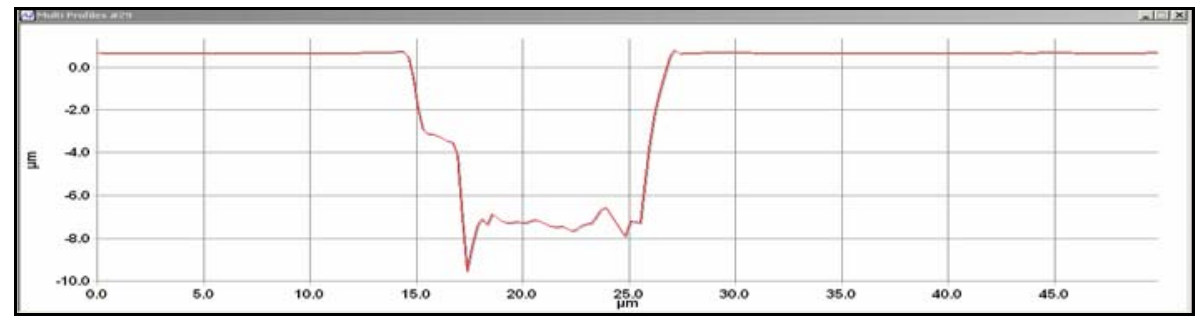

Fig. 6. Depth of Channel (2 Passes) in Quartz, Average Power $59 \mathrm{~mW}$, Pulse Repetition Rate $10 \mathrm{kHz}$, and Laser Beam Traverse Speed of $1 \mu \mathrm{m} / \mathrm{s}$

Since, quartz is transparent to incident laser irradiation at most wavelengths, it is very difficult to machine by the conventional laser micro-machining process. During conventional laser micro-machining, almost all of the incident laser irradiation passes through the quartz workpiece with a very negligible portion being absorbed. Quartz is also a non-conductive material and cannot be machined using the $\mu$-EDM process. Since, in LIP-MM the plasma physically interacts with the target material, the problem of negligible absorption is not encountered.

Single pass and multiple pass channels were made in a quartz workpiece by the LIP-MM process at different peak power density levels and laser beam traverse speeds. Figure 6 shows the depth of a typical multiple pass channel made in quartz, measured through an interferometer (Zygo NewView 7300). The dependence of the depth of a single pass channel on laser beam traverse speed at different peak power density values in quartz is depicted in Fig. 7. It can be seen that irrespective of the peak power density level, significant reduction in channel depth occurs with an increase in the laser beam traverse speed. 


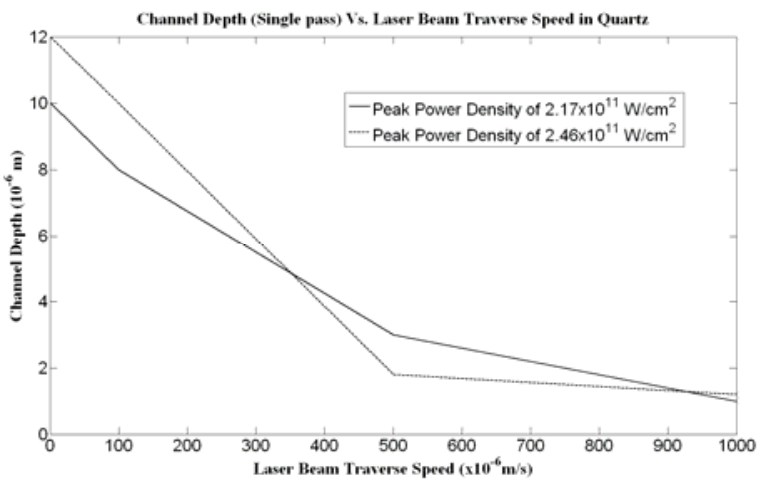

Fig. 7. Dependence of Channel Depth (Single Pass) in Quartz on Laser Beam Traverse Speed at Different Peak Power Density Levels

\section{Results and Discussion}

The breakdown threshold for water corresponding to $10 \%$ plasma occurrence probability was found to be equal to $1.10 \times 10^{11} \mathrm{~W} / \mathrm{cm}^{2}$. The peak power density depends on the average power, pulse repetition rate, pulse duration, and the focal spot size. With 8 ps pulses and a diffraction limited spot size of $21 \mu \mathrm{m}$, a strong dependence of the plasma occurrence probability and its intensity on the peak power density was observed. At near threshold values of the peak power density at all pulse repetition rates, the plasma intensity decreased significantly and could not be observed by the CCD camera. However, no systematic dependence between the breakdown threshold and the pulse repetition rate was observed.

Channels were machined in steel and quartz at different values of peak power densities, laser beam traverse speeds and pulse repetition rates. At a peak power density level corresponding to $100 \%$ plasma occurrence probability, significant recast layer was observed in the micro-machined channels. Irrespective of the pulse repetition rate, recast layer was also observed at very low laser beam traverse speeds, such as $1 \mu \mathrm{m} / \mathrm{s}$.

The best quality surface features with a minimal recast layer, uniform depth and vertical walls was observed when channels were machined in multiple passes at peak power density levels corresponding to $50 \%-70 \%$ of plasma occurrence probability and laser beam traverse speeds corresponding to a few $100 \mu \mathrm{m} / \mathrm{s}$. The channel depth varied significantly with the variation in the laser beam traverse speed. At lower values of the laser beam traverse speed, e.g., $100 \mu \mathrm{m} / \mathrm{s}$, the depth was approximately $30 \mu \mathrm{m}$ at all pulse repetition rates. However, with an increase in the laser beam traverse speed to values such as $500 \mu \mathrm{m} / \mathrm{s}$, the channel depth was found to significantly decrease.

\section{Conclusion}

The feasibility of a new micro-machining process called Laser-Induced Plasma Micro-Machining has been demonstrated. The limitations of $\mu$-EDM and of conventional 
ultra-short laser micro-machining are not observed with the LIP-MM process. Hence, the LIP-MM process attempts to provide a plausible alternative to the many existing limitations associated with both the $\mu$-EDM and ultra-short laser micro-machining processes.

The LIP-MM process is a very novel process for material removal at the microscale. It removes material by plasma-matter interaction. As a matter of fact, it can be postulated that the material removal regime in LIP-MM is very similar to that in $\mu$ EDM. The LIP-MM process also helps in reducing the costs associated with electrode manufacture and dressing in $\mu$-EDM. Finally, it also has the capability to micromachine materials ranging from quartz which is non-conductive and transparent to metals such as steel, nickel, aluminum, etc.

\section{References}

[1] Pham, D.T., Dimov, S.S., Bigot, S., Ivanov, A., Popov, K.: Micro-EDM-Recent Developments and Research Issues. Journal of Materials Processing Technology 149, 50-57 (2004)

[2] Ho, K.H., Newman, S.T.: State of the Art Electrical Discharge Machining (EDM). International Journal of Machine Tools and Manufacture 43, 1287-1300 (2003)

[3] Yu, Z.Y., Kozak, J., Rajurkar, K.P.: Modeling and Simulation of Micro EDM Process. CIRP Annals - Manufacturing Technology 52, 143-146 (2003)

[4] Momma, C., Nolte, S., Chichkov, B.N., Alvensleben, F.v., Tünnermann, A.: Precise Laser Ablation with Ultrashort Pulses. Applied Surface Science 109-110, 15-19 (1997)

[5] Liu, X., Du, D., Mourou, G.: Laser Ablation and Micromachining with Ultrashort Laser Pulses. IEEE Journal of Quantum Electronics 33, 1706-1716 (1997)

[6] Noack, J., Vogel, A.: Laser-Induced Plasma Formation in Water at Nanosecond to Femtosecond Time Scales: Calculation of Thresholds, Absorption Coefficients, and Energy Density. IEEE Journal of Quantum Electronics 35, 1156-1167 (1999)

[7] Shirk, M.D., Molian, P.A.: A Review of Ultrashort Pulsed Laser Ablation of Materials. Journal of Laser Applications 10, 18-28 (1998)

[8] Sacchi, C.A.: Laser-Induced Electric Breakdown in Water. Journal of the Optical Society of America B-Optical Physics 8, 337-345 (1991)

[9] Vogel, A., Nahen, K., Theisen, D., Noack, J.: Plasma Formation in Water by Picosecond and Nanosecond Nd:YAG laser Pulses. I. Optical Breakdown at Threshold and Superthreshold Irradiance. IEEE Journal of Selected Topics in Quantum Electronics 2, 847-860 (1996) 